\title{
Inflamación y disfunción endotelial en pacientes con insuficiencia cardiaca crónica
}

\author{
D ouglas G reig ${ }^{1}$, Pablo Castro ${ }^{1}$, Luigi G abrielli ${ }^{1}$, \\ Rodrigo Miranda ${ }^{1}$, H ugo Verdejo $^{1}$, Hernán Alcaíno ${ }^{2}$, \\ Carlos Bustos' ${ }^{2}$, Mario Chiong ${ }^{2}$, Iván G odoy ${ }^{1}$, \\ Rosemarie Mellado², Lorena G arcía², D aniela Salas², \\ Raúl Vivar2, José Luis Vukasovic ${ }^{3}$, Roberto Concepción ${ }^{4}$, \\ G uillermo Díaz-Araya².
}

\section{Inflammation and endothelial dysfunction in patients with chronic heart failure}

Background: In chronic heart failure (CHF), endothelial dysfunction (ED) is a consequence of an imbalance of vascular tone regulating substances. The relationship between ED and inflammation has not been fully investigated. Aim: To assess the association between inflammation and ED in CHF. Material and methods: Forty two patients aged $56 \pm 14$ years $(80 \%$ male) with a CHF in functional capacity II-III (New York Heart Association) and an ejection fraction (EF) $<40 \%$ were consecutively studied. Patients were classified according to the presence or absence of ED, evaluated by reactive vasodilation measured by ultrasound, after brachial artery compression. Circulating levels of highly sensitive $C$ reactive protein (usCRP), tumor necrosis factor $\alpha$ (TNF $\alpha$ ) and interleukin-6 (IL-6) were determined by ELSA. A group of 15 healthy subjects of similar age, were studied as controls. Results: Sixty seven percent of patients had ED. Compared to controls, patients with CHF had higher usCRP $(0.58 \pm 0.4$ and $4.9 \pm 7.1 \mathrm{mg} / \mathrm{dl}$ respectively, $\mathrm{p}<0.01)$ and IL-6 $(1.38 \pm 0.06$ and $3.1 \pm 1.7 \mathrm{mg} / \mathrm{dl}$ respectively, $\mathrm{p}<0.01)$. Compared to patients without ED, patients with CHF and ED had higher levels of usCRP $(3.0 \pm 0.4$ and $6.0 \pm 5.7 \mathrm{mg} / \mathrm{dl}$ respectively, $\mathrm{p}<0.01)$ and TNF $\alpha$ $(0.31 \pm 0.26$ and $1.0 \pm 1.1 \mathrm{pg} / \mathrm{ml}, \mathrm{p}=0.02)$. No differences in IL-6 were found between CHF groups. Conclusions: In CHF patients, the presence of ED was associated with increased levels of inflammatory markers (Rev Méd Chile 2008; 136: 687-93).

(Key words: Endothelium, vascular; Heart failure; Inflammation)

Recibido el 27 de agosto, 2007. Aceptado el 13 de noviembre, 2007.

${ }_{1}^{1}$ Departamento de Enfermedades Cardiovasculares, Pontificia Universidad Católica de Chile. ${ }^{2}$ Facultad de Ciencias Químicas y Farmacéuticas, Universidad de Chile. ${ }^{3}$ Facultad de Medicina, Universidad de Chile. ${ }^{4}$ Hospital DIPRECA, Santiago, Chile.

Correspondencia a: Dr. Pablo Castro. Pontificia Universidad Católica de Chile. Marcoleta 367, Santiago, Chile. E-mail: pcastro@med.puc.cl o Dr. Guillermo Díaz-Araya, Facultad Ciencias Químicas y Farmacéuticas, Universidad de Chile, Olivos 1007, Santiago, Chile. E-mail: gdiaz@ciq.uchile.cl 
$\mathrm{E}^{\mathrm{n}}$ pacientes con insuficiencia cardíaca (IC) y disfunción ventricular izquierda, la disminución de la eficacia contráctil está asociada a un proceso inflamatorio con aumento de citoquinas, activación neurohumoral (sistemas simpático, renina-angiotensina-aldosterona y arginina-vasopresina, entre otros) y estrés oxidativo $(\mathrm{EO})^{1}$. Estudios recientes de pacientes con IC muestran niveles elevados de reactantes de fase aguda, como proteína $\mathrm{C}$ reactiva ultrasensible (PCRus) y citoquinas, tales como, interleuquina 6 (IL-6) y factor de necrosis tumoral alfa (TNF $\alpha)$, lo que se asocia a mayores eventos cardiovasculares y aumento de la morbimortalidad 2,3 .

La función endotelial se altera en enfermedades cardiovasculares, jugando un papel protagónico en la regulación del tono, homeostasis e inflamación vascular ${ }^{4}$. Ya hace algunos años se demostró la presencia de disfunción endotelial (DE) en pacientes con IC y su valor pronóstico ${ }^{5}$. La DE en la IC se ha atribuido a una disminución de la síntesis del óxido nítrico, aumento de su degradación por especies reactivas del oxígeno, inflamación e hiporrespuesta del músculo liso vascular $^{4,6-8}$.

La interacción entre los mecanismos citados es compleja y no está bien definida, pero tienen un efecto adverso en la remodelación y función ventricular, en el tono vascular (expresado como DE) y en el desarrollo de enfermedad aterosclerótica, favoreciendo la progresión de la IC ${ }^{9}$.

Recientemente, se ha descrito en pacientes con enfermedad coronaria una correlación entre PCRus y $\mathrm{DE}$, sugiriendo un nexo entre inflamación y función endotelial en enfermedades cardiovasculares ${ }^{10}$. Sin embargo, esta relación en pacientes con IC no ha sido sistemáticamente evaluada.

En el presente estudio se investigaron los niveles plasmáticos de estos marcadores inflamatorios y la presencia o ausencia de $\mathrm{DE}$ en pacientes con IC.

\section{MÉtodos}

Pacientes y controles. Se estudiaron 42 pacientes consecutivos con IC capacidad funcional (CF) II-III de la New York Heart Association (NYHA), provenientes de nuestros centros ambulatorios. Todos los pacientes firmaron el consentimiento informado aprobado por el Comité de Ética de cada institución participante. Los critenios de inclusión fueron: a) Fracción de eyección del ventrículo izquierdo menor a 40\% determinada por ecocardiografía; b) Tratamiento médico convencional con bloqueadores beta-adrenérgicos, diuréticos e inhibidores de la enzima convertidora 0 antagonistas de los receptores de angiotensina; c) Situación clínica estable durante las últimas cuatro semanas. Se excluyeron aquellos pacientes con: a) Angina inestable, infarto del miocardio, cirugía de revasculanización miocárdica o angioplastia coronaria en los últimos 6 meses; b) Hipertensión arterial no controlada definida como presión arterial sistólica mayor a $140 \mathrm{mmHg}$ o presión arterial diastólica mayor a $90 \mathrm{mmHg}$; c) Miocardiopatía hipertrófica, cardiopatía congénita o enfermedades valvulares significativas y d) Enfermedades concomitantes que afecten las determinaciones de marcadores inflamatorios (infecciones, neoplasias y enfermedades autoinmunes, entre otras).

Se utilizó un grupo control de 15 sujetos sanos de similar distribución en edad y sexo. Ninguno de ellos presentaba factores de riesgo coronario conocidos, terapia farmacológica ni DE.

Evaluación de disfunción endotelial. Luego de 30 min en reposo, se obtuvo mediante ecografía imágenes bidimensionales de la arteria braquial del brazo derecho, en estado basal y luego de $5 \mathrm{~min}$ de hiperemia reactiva inducida por oclusión con manguito (observador único, transductor 7-11 MHz, Sonos 5500, Philips) $)^{11,12}$. Los resultados se expresaron como porcentaje de cambio en el diámetro de la arteria. La DE dependiente del endotelio se definió como un porcentaje de cambio menor a $7 \%$.

Evaluación de los marcadores inflamatorios sistémicos. Los niveles plasmáticos de TNF $\alpha$, PCRus e IL-6 se determinaron mediante ensayos disponibles comercialmente (ELISA). Para el caso de la PCRus se utilizó un ELISA de competencia (Calbiochem-Novabiochem).

Estadística. Los grupos fueron testeados para normalidad mediante test de Shapiro-Wilk. Los grupos se compararon mediante el test de MannWhitney U para variables continuas y test de chi cuadrado para la comparación de variables categóricas. Además, para la comparación de tertiles de IL-6, TNF $\alpha$ y PCRus se utilizó el test de 
Spearman para correlación de variables ordinales. Los resultados se expresaron como promedio \pm desviación estándar (SEM). Un valor $\mathrm{p}<0,05$ se consideró significativo.

\section{RESULTADOS}

La edad promedio fue $56 \pm 14$ años (rango 20 a 85), 34 hombres (80\%), 28 pacientes $(67 \%)$ presentaron DE. La Tabla 1 muestra las características de los pacientes con IC según presencia o ausencia de DE. En el grupo control, la edad promedio fue de $56 \pm 15$ años, $80 \%$ fueron hombres y el porcentaje de cambio endotelio dependiente fue de $10 \% \pm 1,5 \%$. El porcentaje de cambio endotelio dependiente en el grupo global de pacientes con IC fue de $4,4 \% \pm 1,9 \%$, siendo de $3,7 \% \pm 2,1 \%$ en los pacientes con IC y DE, mientras que en aquellos sin DE fue de $11,1 \pm 1,6$ (valor $\mathrm{p}<0,001$ ). Respecto a los marcadores de inflamación, los pacientes con IC presentaron nive- les aumentados de PCRus e IL-6 respecto del grupo

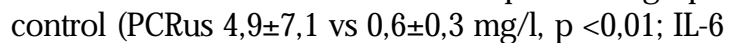
$3,1 \pm 1,7$ vs $1,4 \pm 0,1 \mathrm{mg} / \mathrm{dl}, \mathrm{p}<0,01)$. Se observó una tendencia no significativa a mayores valores de TNF $\alpha$ en el grupo de sujetos con IC respecto del grupo control $(0,8 \pm 0,9$ vs $0,5 \pm 0,3 \mathrm{pg} / \mathrm{ml})$. Los pacientes con IC y DE presentaron niveles elevados de PCRus y TNF $\alpha$ respecto de los pacientes con IC sin DE (PCRus 6, $0 \pm 5,7$ vs 3,0 $00,4 \mathrm{mg} / \mathrm{dl}, \mathrm{p}<0,01$; TNF $\alpha$ $1,0 \pm 1,1$ vs $0,3 \pm 0,3 \mathrm{pg} / \mathrm{ml}, \mathrm{p}=0,02)$. No hubo diferencias en los niveles de IL-6 respecto a la presencia de DE en los pacientes con IC. La Figura 1 muestra que en el grupo con $\mathrm{DE}$, la proporción de pacientes con CF III fue de 65\%, mientras que en el grupo sin DE fue de $21 \%$ (valor $p=0,02$ ). Las Figuras 2 y 3 muestran la distribución de pacientes agrupados por tertiles de TNF $\alpha$ y PCRus según presencia 0 ausencia de DE. El $71 \%$ de los pacientes con DE tenían valores plasmáticos de TNF $\alpha$ de $0,22 \mathrm{pg} / \mathrm{ml}$ vs $46 \%$ de los pacientes sin DE ( $r=0,35$, valor $\mathrm{p}=0,02$ para la comelación). Además, $79 \%$ de los pacientes

Tabla 1. Características de los pacientes con IC de acuerdo a la función endotelial

\begin{tabular}{|c|c|c|c|}
\hline & Con DE $(\mathrm{n}=28)$ & $\operatorname{Sin} \mathrm{DE}(\mathrm{n}=14)$ & Valor $\mathrm{p}$ \\
\hline \multicolumn{4}{|l|}{ Edad, años } \\
\hline (promedio \pm SEM) & $57 \pm 16$ & $56 \pm 9$ & 0,9 \\
\hline Sexo masculino-n (\%) & $23 \quad(82)$ & $11(79)$ & 0,5 \\
\hline Etiología de la IC-n (\%) & & & $0,5^{\llbracket}$ \\
\hline Hipertensiva & $5 \quad(18)$ & $2(14)$ & \\
\hline Isquémica & $6 \quad(21)$ & $6(42)$ & \\
\hline Idiopática & $13(46)$ & $5(36)$ & \\
\hline Otra & 4 (14) & $1(7)$ & \\
\hline \multicolumn{4}{|l|}{ Factores de riesgo-n (\%) } \\
\hline Hipertensión arterial & $16(57)$ & $7(50)$ & 0,5 \\
\hline Diabetes mellitus & 4 (14) & $2(14)$ & 0,3 \\
\hline Dislipidemia & $4 \quad(14)$ & $3(21)$ & 0,4 \\
\hline Tabaquismo & $5 \quad(18)$ & $2(14)$ & 0,6 \\
\hline \multicolumn{4}{|l|}{ Tratamiento n (\%) } \\
\hline IECA o ARA II & $18(64)$ & $12(86)$ & 0,14 \\
\hline Betabloqueadores & $23 \quad(82)$ & $12(86)$ & 0,6 \\
\hline Diuréticos & $23(82)$ & $12(86)$ & 0,6 \\
\hline Espironolactona & 22 (79) & $11(79)$ & 0,7 \\
\hline FEVI\% & $29 \pm 5,5$ & $31 \pm 3,3$ & 0,4 \\
\hline
\end{tabular}

DE: Disfunción endotelial. IECA: Inhibidor de enzima convertidora de angiotensina I. ARA II: inhibidores del receptor de angiotensina II. FEVI: fracción de eyección de ventrículo izquierdo.

I: Para el grupo completo. 


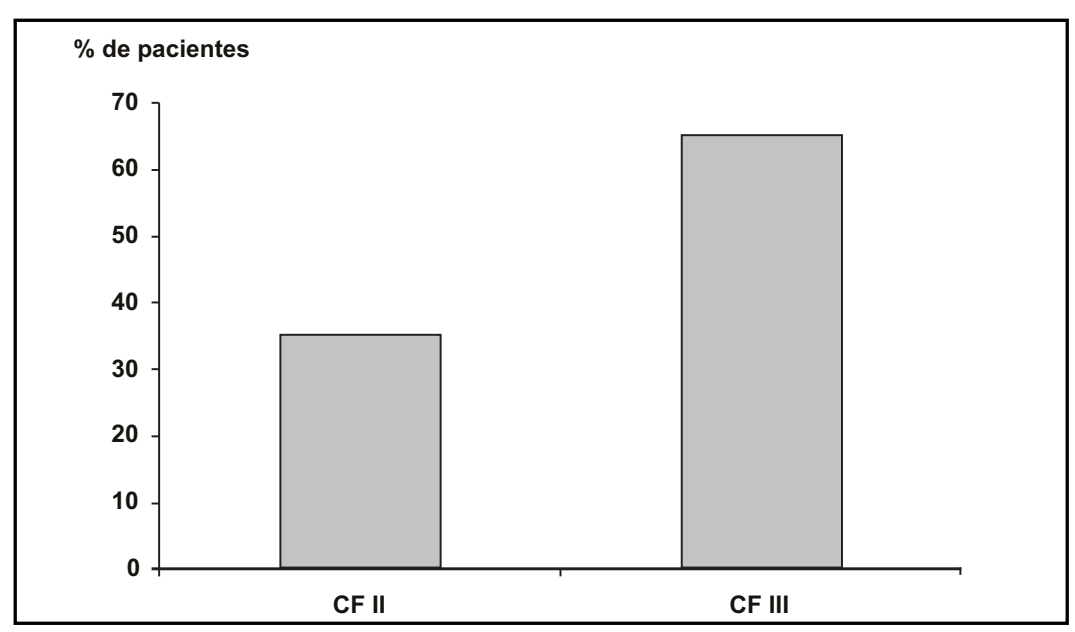

Figura 1. Distribución de pacientes con DE según clase funcional de la NYHA. Valor $\mathrm{p}<0,05$.

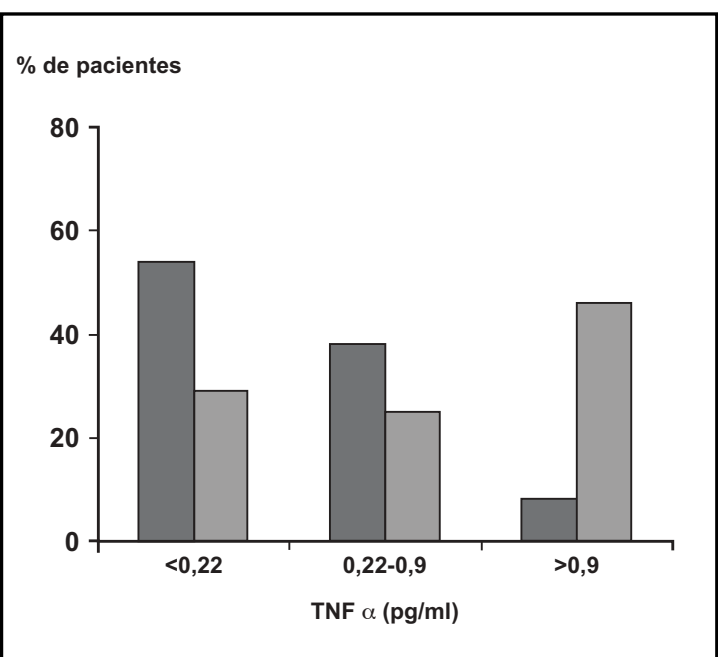

Figura 2. Proporción de pacientes con (barra gris) y sin (barra negra) disfunción endotelial según tertiles de niveles circulantes del factor de necrosis tumoral alfa $(\mathrm{TNF} \alpha)$. $\mathrm{R}=0,353$, valor $\mathrm{p}=0,02$ para la comelación.

con DE tenían niveles circulantes de PCRus $\geq 3,6 \mathrm{mg} /$ $\mathrm{dl}$ vs $50 \%$ de los pacientes sin $\mathrm{DE}(\mathrm{r}=0,34, \mathrm{p}=0,03$ para la correlación). Los niveles de IL-6 no fueron diferentes en los pacientes con y sin $\mathrm{DE}(3,3 \pm 1,8$ vs $2,2 \pm 1,2 \mathrm{pg} / \mathrm{ml}$, respectivamente, $\mathrm{p}=0,12$ ). No se encontraron diferencias en los valores de PCRus, TNF $\alpha$ o IL-6 entre pacientes de IC de diferentes etiologías (datos no mostrados).

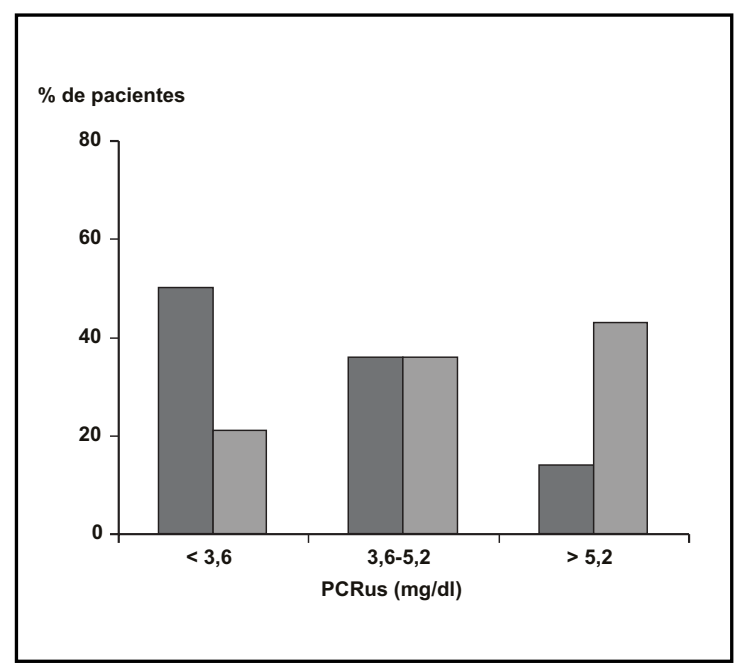

Figura 3. Proporción de pacientes con (barra gris) y sin (barra negra) disfunción endotelial según tertil de nivel plasmático de proteína $\mathrm{C}$ reactiva ultrasensible (PCRus). $\mathrm{r}$ $=0,336$, valor $\mathrm{p}=0,03$ para la comelación.

\section{Discusión}

El principal hallazgo de este estudio es que en pacientes con IC, la presencia de DE se asoció a presencia de factores proinflamatorios determinada por PCRus y citoquinas inflamatorias (IL-6, $\mathrm{TNF} \alpha$ ) y a un mayor deterioro de la capacidad funcional de la NYHA. Los pacientes con IC 
presentaron niveles elevados de PCRus e IL-6, respecto del grupo control.

En pacientes con IC, la presencia de DE se ha asociado a un pronóstico desfavorable y mayor mortalidad $^{5,13}$. En pacientes con IC de etiología isquémica, el exceso de mortalidad podría estar relacionado con aumento de los eventos isquémicos. Sin embargo, otros mecanismos también contribuyen, ya que esta asociación parece ser independiente de la etiología de la IC. La DE en la microcirculación coronaria podría inducir áreas de isquemia o necrosis en ausencia de enfermedad ateroesclerótica. Adicionalmente, por cambios en las condiciones de carga ventricular se promueve remodelamiento ventricular y progresión de la enfermedad.

El endotelio mantiene el tono vascular mediante un estricto balance entre sustancias vasodilatadoras y vasoconstrictoras. Además, regula la coagulación y participa en la inflamación, mediante la producción de citoquinas proinflamatorias y la expresión de moléculas de adhesión ${ }^{13}$. La fisiopatología de la $\mathrm{DE}$ es compleja y no es exclusiva de la IC, por ejemplo, se ha descrito DE en pacientes con hipertensión arterial, diabetes mellitus y ateroesclerosis ${ }^{14-16}$.

Para evaluar la función endotelial, nosotros utilizamos el modelo de vasodilatación reactiva postcompresión ${ }^{11,12}$. Este método se ha utilizado para validar indirectamente la presencia de DE en diferentes territorios vasculares. Modelos humanos han determinado que es mediado por la liberación de óxido nítrico, prostaglandinas y activación simpática, entre otros ${ }^{17,18}$.

Por otro lado, estudios recientes han establecido la importancia de la activación del sistema inmune y la inflamación en la patogénesis de la $\mathrm{IC}^{1}$. Las citoquinas inflamatorias pueden modular la función cardiovascular a través de diferentes mecanismos, tales como alteración en la homeostasis del calcio, disminución del inotropismo a través de la alteración de receptores betaadrenérgicos y regulación de la hipertrofia y apoptosis cardíaca ${ }^{19,20}$. Del mismo modo, diversos estudios han relacionado a la DE con niveles aumentados de TNF $\alpha$ e IL-6; sin embargo, su asociación con PCRus no es clara. La IL-6 se ha relacionado a mal pronóstico, determinándose en el estudio de Framingham como un factor independiente de mortalidad $^{21}$. La IL-6 es producida por el sistema monocito/macrófago, células endoteliales, fibroblastos y células musculares lisas y cardiacas en respuesta a múltiples estímulos, como la hipoxia ${ }^{22,23}$. Además, algunos reportes han mostrado que su aumento es mayor en pacientes con IC de etiología isquémica comparados con miocardiopatía dilatada 24 . Pese a lo anterior, el papel de la IL-6 en la patogenia de la IC es compleja, ya que evidencia reciente sugiere que su aumento sería un factor cardioprotector al promover la neovascularización e hipertrofia compensatoria ${ }^{25}$. En nuestro trabajo, no se encontró una relación entre IL-6 y DE. Tampoco hubo diferencias entre pacientes con IC de etiología isquémica vs no isquémica. Además, los niveles de IL-6 fueron menores a los descritos en la literatura ${ }^{26}$, lo que cual podría deberse a una situación clínica más estable de la población estudiada. Por otro lado, se han detectado niveles circulantes aumentados de TNF $\alpha$ en diferentes estadios de pacientes con IC, siendo mayor su aumento en las etapas más avanzadas de la IC, independiente de su etiología ${ }^{27}$. La principal fuente de TNF $\alpha$ son los macrófagos circulantes y adheridos al endotelio ${ }^{28}$. Diversas terapias en humanos han intentado antagonizar su acción con resultados desalentadores en esta área, lo que señala que TNF $\alpha$ no es la única citoquina responsable de la progresión de la enfermedad $^{29}$. Modelos animales han establecido que actuaría mediante la activación del factor nuclear kappa B y que su inhibición podría relacionarse con disminución del tamaño del infarto inducido experimentalmente ${ }^{30}$. Nuestros hallazgos concuerdan con los publicados por Tentolourius et $\mathrm{al}^{24}$, en los cuales se observó un aumento de TNF $\alpha$ en pacientes con IC de cualquier etiología y la correlación de sus niveles plasmáticos con el grado de DE. Su relación de causalidad escapa al modelo planteado en este estudio.

La PCR es una proteína de fase aguda sintetizada en el hígado como respuesta principalmente, a la IL-6 ${ }^{22}$. Diversos estudios han mostrado que es un predictor sensible de eventos cardiovasculares adversos $^{26}$, incluso en individuos sanos, quienes tienen, habitualmente, valores $\leq 2 \mathrm{mg} / \mathrm{dl}^{26,31}$. Su mecanismo de daño en la IC es complejo, involucrando, por mencionar algunos de sus efectos, la activación del complemento que conlleva a apoptosis, DE mediada por inactivación de óxido nítri- 
co $^{32}$ e inducción de otras citoquinas y moléculas de adhesión ${ }^{33}$. Además, se ha postulado un efecto proinflamatorio directo en las células endoteliales $^{34}$. La principal diferencia entre PCR y PCRus radica en que ésta tiene un rango de detección más bajo, lo que la hace más sensible en la estratificación del riesgo cardiovascular ${ }^{26}$. No hay estudios que vinculen PCRus y el grado de DE en pacientes con IC. En nuestro estudio, al igual como se observa con $\mathrm{TNF} \alpha$, los niveles plasmáticos de PCRus fueron mayores en el grupo con DE y valores elevados ( $\geq 5,2 \mathrm{mg} / \mathrm{dl})$ se asociaron a una mayor proporción de pacientes con DE.

Nuestro estudio muestra observaciones y correlaciones en una población seleccionada de pacientes estables y con tratamiento optimizado de la IC, lo cual limita la extrapolación de los resultados a la población general de pacientes con IC. Por otro lado, nuestro estudio evalúa un número reducido de pacientes y el modelo impide demostrar causalidad. El grupo control no tenía factores de riesgo conocidos, sin embargo, no existió diferencias en los factores de riesgo entre el grupo de pacientes

\section{REFERENCIAS}

1. Yndestad A, Kristian DJ, Oie E, Ueiand T, GuLestad L, AuKRUST P. Systemic inflammation in heart failurethe whys and wherefores. Heart Fail Rev 2006; 11: 83-92.

2. Bieske BE, Nickias JM, Bard RL, Brook RD, Gurbel PA, BLDEN KP ET AL. Neutral effect on markers of heart failure, inflammation, endothelial activation and function, and vagal tone after high-dose HMGCoA reductase inhibition in non-diabetic patients with non-ischemic cardiomyopathy and average low-density lipoprotein level. J Am Coll Cardiol 2006; 47: 338-41.

3. Huang PH, Leu HB, Chen JW, Wu TC, Lu TM, Ding YA ET AL. Comparison of endothelial vasodilator function, inflammatory markers, and N-terminal pro-brain natriuretic peptide in patients with or without chronotropic incompetence to exercise test. Heart 2006; 92: 609-14.

4. Widlansky ME, Gokce N, Keaney JF JR, Vita JA. The clinical implications of endothelial dysfunction. J Am Coll Cardiol 2003; 42: 1149-60.

5. Katz SD, Hryniewicz K, Hriljac I, Balddemaj K, Dimayuga C, Hudaihed A ET al. Vascular endothelial con y sin DE. En el presente estudio no se exploraron otros marcadores (i.e: células endoteliales periféricas, células progenitoras endoteliales entre otras ${ }^{35}$ ) que pudiesen estar alterados en pacientes con IC. Del mismo modo, el hecho que los coeficientes de determinación entre TNF $\alpha$ y DE, así como entre PCRus y DE sean significativos, pero relativamente bajos $\left(\mathrm{r}^{2}=12 \%\right)$, señalan que otros factores participan en la etiopatogenia de la $\mathrm{DE}^{4}$.

En resumen, los pacientes con IC y DE estables y en tratamiento médico optimizado tienen valores aumentados de citoquinas inflamatorias, principalmente TNF $\alpha$, y PCRus. Estos hallazgos fortalecen la asociación entre inflamación medida a través de marcadores en sangre periférica y DE en pacientes con IC. Estrategias destinadas a disminuir estos marcadores inflamatorios, podrían contribuir a mejorar la función endotelial en pacientes con IC y DE.

\section{Agradecimientos}

Este trabajo fue financiado en parte por el Proyecto FONDECYT 1050768.

dysfunction and mortality risk in patients with chronic heart failure. Circulation 2005; 111: 310-14.

6. Tousouls D, CharaKida M, Stefanadis C. Inflammation and endothelial dysfunction as therapeutic targets in patients with heart failure. Int J Cardiol 2005; 100: 347-53.

7. Miranda R, Castro P, Verdejo H, Chiong M, DíazAraya G, Melado R. Estrés oxidativo e inflamación en Insuficiencia Cardíaca: Mecanismos de daño y alternativas terapéuticas. Rev Méd Chile 2007; 135: 1056-63.

8. LANDMESSER U, DREXIER $H$. The clinical significance of endothelial dysfunction. Curr Opin Cardiol 2005; 20: 547-51.

9. CoRRY DB, Tuck ML Uric acid and the vasculature. Curr Hypertens Rep 2006; 8: 116-9.

10. PeterSEN JW, FetKeR GM. Inflammatory biomarkers in heart failure. Congest Heart Fail 2006; 12: 324-8.

11. Ceifrmajer DS, Sorensen KE, Gooch VM, Spiegelhalter DJ, Muler OI, SuLvan ID et al. Non-invasive detection of endothelial dysfunction in children and adults at risk of atherosclerosis. Lancet 1992; 340: 1111-5.

12. Corretti MC, Anderson TJ, Benjamin Ej, Ceiermager D, Charbonneau F, Creager MA et al. Guidelines for the 
ultrasound assessment of endothelial-dependent flow-mediated vasodilation of the brachial artery: a report of the International Brachial Artery Reactivity Task Force. J Am Coll Cardiol 2002; 39: 257-65.

13. LiBBY P, RIDKER PM, MASERI A. Inflammation and atherosclerosis. Circulation 2002; 105: 1135-43.

14. Panza JA, Quyyumi AA, Brush JE Jr, Epstein SE. Abnormal endothelium-dependent vascular relaxation in patients with essential hypertension. NEngl J Med 1990; 323: 22-7.

15. Beckman JA, Goddine AB, Dunaif A, Gerhard-Herman M, CReager MA. Endothelial Function Varies According to Insulin Resistance Disease Type. Diabetes Care. On line, Jan 29, 2007.

16. Zeiher AM, Drexier $H$, Wolschiager $H$, Just $H$. Endothelial dysfunction of the coronary microvasculature is associated with coronary blood flow regulation in patients with early atherosclerosis. Circulation 1991; 84: 1984-92.

17. JoANNides R, HaeFeu WE, LNDER L, Richard V, BAKKAL EH, Thuilez C ET AL. Nitric oxide is responsible for flow-dependent dilatation of human peripheral conduit arteries in vivo. Circulation 1995; 91: 13149.

18. Sun D, Huang A, Smith CJ, Stackpole CJ, Connetta JA, SHESEIY J ET AL. Enhanced release of prostaglandins contributes to flow-induced arteriolar dilation in eNOS knockout mice. Circ Res 1999; 85: 288-93.

19. Yokoyama T, Vaca L, Rossen RD, Durante W, Hazarika P, MANN DL Cellular basis for the negative inotropic effects of tumor necrosis factor-alpha in the adult mammalian heart. J Clin Invest 1993; 92: 2303-12.

20. Finkel MS, Oddis CV, Jacob TD, Watkins SC, HatTLER BG, SImmons RL Negative inotropic effects of cytokines on the heart mediated by nitric oxide. Science 1992; 257: 387-9.

21. Vasan RS, Sumvan LM, D'agostino RB, Roubenofy $R$, HARRIS T, SAWYER DB ET AL. Inflammatory markers and risk of heart failure in elderly subjects without prior myocardial infarction: the Framingham Heart Study. Circulation 2003; 107: 1486-91.

22. Sato $Y$, Takatsu $Y$, Kataoka $K$, Yamada T, Taniguchi $R$, SASAYAMA $S$ ET AL. Serial circulating concentrations of C-reactive protein, interleukin (IL)-4, and IL-6 in patients with acute left heart decompensation. Clin Cardiol 1999; 22: 811-3.

23. Yamauchi-Takihara $K$, Ihara $Y$, Ogata A, Yoshizaki $K$, Azuma J, Кishimoto T. Hypoxic stress induces cardiac myocyte-derived interleukin-6. Circulation 1995; 91: 1520-4.

24. Tentolouris $C$, Tousouns $D$, Antoniades $C$, Bosinakou E, Kotsopoulou M, TriKas A et al. Endothelial function and proinflammatory cytokines in patients with ischemic heart disease and dilated cardiomyopathy. Int J Cardiol 2004; 94: 301-5.

25. HilfiKer-KieINer D, LANDMESSER U, DreXier H. Molecular mechanisms in heart failure focus on cardiac hypertrophy, inflammation, angiogenesis, and apoptosis. J Am Coll Cardiol 2006; 48: A56-A66.

26. Yin WH, Chen JW, Jen HL, Chiang MC, Huang WP, FENG AN ET AL. Independent prognostic value of elevated high-sensitivity C-reactive protein in chronic heart failure. Am Heart J 2004; 147: 931-8.

27. Levine B, Kalman J, Mayer L, Fimt HM, Packer M. Elevated circulating levels of tumor necrosis factor in severe chronic heart failure. N Engl J Med 1990; 323: 236-41.

28. VASSAW P. The pathophysiology of tumor necrosis factors. Annu Rev Immunol 1992; 10: 411-52.

29. Von HS, JankowSKa EA, ANKER SD. Tumour necrosis factor-alpha and the failing heart-pathophysiology and therapeutic implications. Basic Res Cardiol 2004; 99: 18-28.

30. Wong SC, Fukuchi M, MeINYK P, Rodger I, Giaid A. Induction of cyclooxygenase-2 and activation of nuclear factor-kappaB in myocardium of patients with congestive heart failure. Circulation 1998; 98: 100-3.

31. GABAY C, KUSHNER I. Acute-phase proteins and other systemic responses to inflammation. N Engl J Med 1999; 340: 448-54.

32. Ciark DJ, Cleman MW, Pfau SE, Rowins Sa, Ramahi TM, MAYER C ET AL. Serum complement activation in congestive heart failure. Am Heart J 2001; 141: 684-90.

33. Y In WH, Chen JW, Jen HL, Chiang MC, Huang WP, Feng AN ET AL. The prognostic value of circulating soluble cell adhesion molecules in patients with chronic congestive heart failure. Eur J Heart Fail 2003; 5: 507-16.

34. Pasceri V, WiLerson JT, YeH ET. Direct proinflammatory effect of C-reactive protein on human endothelial cells. Circulation 2000; 102: 2165-8.

35. Deanfield John E, Halcox Julan P, Rabelnk Ton J. Endothelial Function and Dysfunction: Testing and Clinical Relevance. Circulation 2007; 115: 1285-95. 